\title{
Numerical testing of steel beam-to-column bolted extended end-plate connection with haunches
}

\author{
Dominiq Jakab ${ }^{1,}{ }^{*}$, Aurel Stratan ${ }^{1}$, and Dan Dubina ${ }^{1,2}$ \\ 1Politehnica U niversity of Timisoara, Department of Steel Structures and Structural M echanics, Ioan \\ Curea nr. 1, Timisoara, Romania \\ ${ }^{2}$ Romanian A cademy, Timisoara Branch, M ihai V iteazu nr. 24, Timisoara, Romania
}

\begin{abstract}
During the European research project entitled EQUALJ OINTS (European pre-Qualified steel JOINTS) [1], which recently concluded, the matter of providing a set of pre-qualification procedures for moment resisting beam-to-column connections which are currently used in Europe has been addressed. During the experimental campaign 24 specimens with bolted extended end-plate connections with haunches were tested. The current paper presents the numerical model which has been developed such that numerical testing may be performed to further investigate specific details. In what concerns the material, an isotropic material model has been calibrated and used based on tensile tests of coupons extracted from the specimens to model the actual plastic behaviour. Moreover, the imperfections of the beam have been taken into account using a bucking analysis to model as accurate as possible the specimens tested. The interaction between parts has been modelled using contacts with different interaction laws. The model used for the bolt has been calibrated such that the prel oading and failure replicate the behaviour of the actual bolts used in the experiments.
\end{abstract}

\section{INTRODUCTION}

The use of pre-qualified moment resisting joints in steel structures is a common practice in U.S and Japan. The guidelines for the design of connections cannot be adopted in Europe, owing to the different typologies of the joints, sections of the elements and materials. Consequently, the European research project entitled EQUALJOINTS [1] has provided a set of pre-qualification criteria for the most used joint typologies in Europe. During the project, a large experimental campaign has been conducted in which 24 specimens with bolted extended end-plate connections with haunches have been tested.

The current paper presents the numerical model which was developed and calibrated for further investigations on specific details. The modelling of the material and bolts as well as the influence of the type of finite element and imperfections are displayed. The calibration

\footnotetext{
${ }^{*}$ Corresponding author: dominiq.jakab@ student.upt.ro
} 
of the joint started on sub-assemblies for material and bolts, while the particularities of the joint model were adjusted based on the monotonic experimental tests.

\section{Numerical model}

\subsection{Generalities}

The experimental campaign for the bolted connections with haunches focused on the behaviour of the joint under monotonic and cyclic loading. Specific details on the experimental tests are available in [2]. The numerical simulations were performed with the finite element software Abaqus [3]. The numerical model which was chosen is presented in Fig. 1.
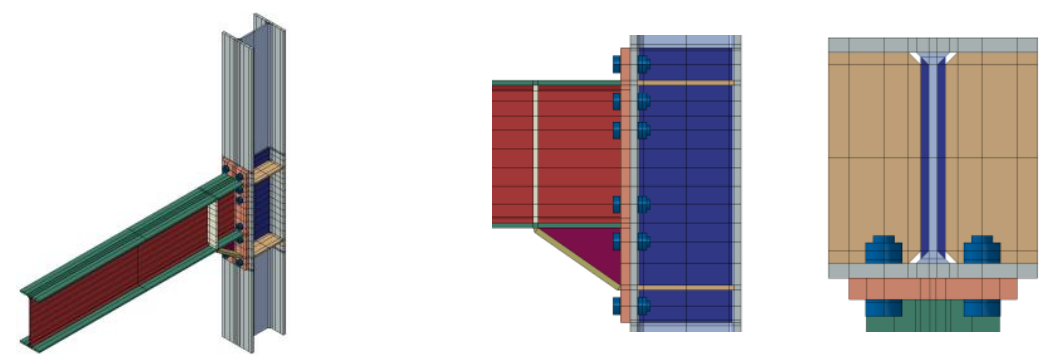

Fig. 1. Finite element model of a single sided joint with side and top views

Solid elements were chosen to model all parts of the joint due to the complex geometry. The measured geometry of the specimens from the experimental tests has been used for modelling. As solvers, a Dynamic Implicit with the quasi-static option was preferred for the analyses. Tie constraints were used for the welded parts of the connections. The interaction between the end-plate and column flange was defined using a hard contact with penalty for normal behaviour and penalty with friction $(0.3$ for the friction coefficient) for tangential behaviour.

The column is loaded at the tip in displacement control. The supports at the bottom of the column, respectively the links which are located at the end of the beam are considered as pinned. However, due to the fact that the failure mechanism is concentrated mainly in the beam leading to buckling of the parts in compression, some lateral restraints were used to avoid the out-of-plane movements. Consequently, continuum distributing coupling constraints were used to simulate the points were the beam was restrained laterally. As boundary conditions, the displacement of that points was prevented out of plane.

Considering the solver from Standard Abaqus library, the preload applied to the bolts was performed using a bolt load. In addition, the interaction law which simulates the behaviour until failure of the bolts is presented in detail in chapter 2.3.

\subsection{Material model}

Two sets of coupons (one from the factory, one extracted from the tested specimens) provided data from the most important components of the joint: beam flange and web, haunch flange and web and end-plate. The properties obtained from the tensile tests are: tensile strength $\left(R_{\mathrm{m}}\right)$, proof test $\left(R_{\mathrm{p} 0.2}\right)$, upper yield strength $\left(R_{\mathrm{eH}}\right)$ and percentage elongation after fracture $(A)$. Using these properties an engineering stress-strain curve was computed as an average curve. For the numerical simulations, the true stress-true strain curves were determined based on the engineering curve, using the provisions from [4]. 
The formulae provided in [4] are valid only until $R_{\mathrm{m}}$. Then, trial values were used for stress and strain to simulate the material failure. For this procedure, numerical simulations of the coupons were performed in Abaqus software - Fig. 2 a). The finite element C3D8I was used. Due to the fact that the stress-strain curves are mesh dependent, the same finite element, dimension and mesh configuration used on the numerical simulation of the joint is also used for the material calibration. To model the plastic behaviour, material plasticity with isotropic hardening was used, considering only monotonic loading on the joint. In Fig. 2 a) is presented the calibration of a material for a certain element in which the results from numerical simulation is compared to the average engineering curve.

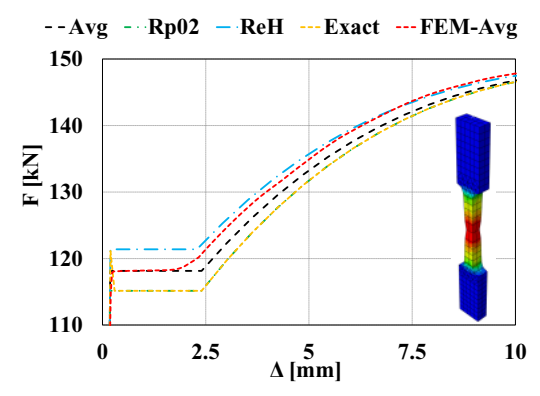

a)

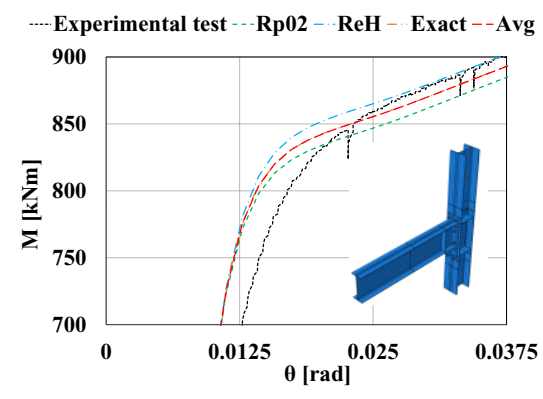

b)

Fig. 2. Material calibration: a) Calibration on coupon, b) Calibration on joint

Different yield limits were chosen $\left(R_{\mathrm{p} 0.2}, R_{\mathrm{eH}}\right.$ and an average between the two to replicate a real material model, which reaches first $R_{\mathrm{eH}}$, then decreases to $R_{\mathrm{p} 0.2}$ and increasing again - curve named Exact in Fig. 2 a)) to model the material. In Fig. 2 b) are presented the results in terms of moment rotation which show the influence of different yield limits on a joint model. Based on these results and on the computational time, it was decided that the most appropriate material model is the one which considers the average between $R_{\mathrm{eH}}$ and $R_{\mathrm{p} 0.2}$ as yield limit.

\subsection{Bolt model}

Regarding the material for the bolt, the nominal values obtained from [5] were used. The same procedure from chapter 2.2 for obtaining the true stress-true strain curve has been applied. Similar to the tensile tests, within the project, tests for M27 and M30 grade bolts were performed, with different lengths of the shank. As output data, the final experimental curve (F- $\Delta$ ) was obtained using the procedures provided in [5]. The bolts are all HV, which means that the failure of the bolts should be in the threaded zone, such that the bolt is more ductile.

In literature are presented several FE models which may be adopted for the bolt. In [6] and [7] a very detailed FE model has been considered in which the entire geometry is modelled. However, for this application such a model is not appropriate due to the large number of finite elements. Consequently, a simpler model has been chosen, similar to the one adopted in [8]. Anyway, the model presented in the current article with the connector linked in series with the bolt shank (see Fig.3) has the advantage that not only the axial connector but also the bolt shank can exhibit axial stresses compared to the one adopted in [8]. The axial connector used is characterized by a force-displacement feature which was assigned to a wire, linked between the bolt shank and the bolt nut. The displacement used to characterize the nonlinear behaviour $\left(\Delta_{\mathrm{th}}\right)$ were obtained by subtracting the equivalent 
elastic displacement $\left(\Delta_{\mathrm{e}}\right)$ of the shank from the total displacement determined in the experimental tests $-\Delta$. The procedure is presented in Fig. 4 .

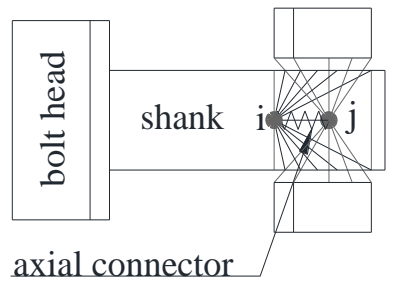

Fig. 3. Bolt model

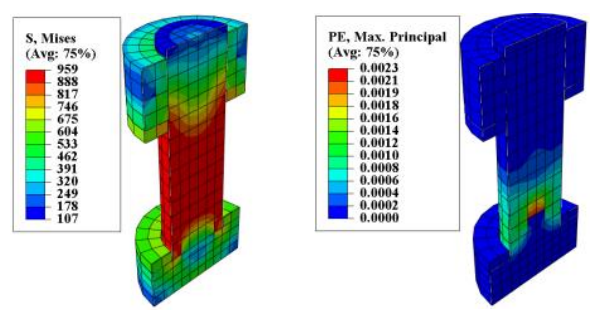

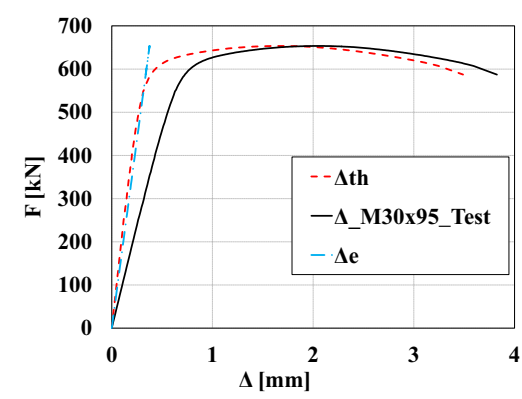

Fig. 4. Procedure for determining non-linear bolt law

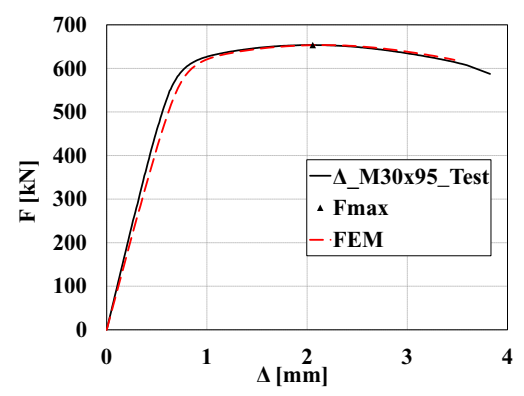

Fig. 6. Bolt model calibration

Fig. 5. Equivalent Von Mises Stress at maximum force in the bolt

In the numerical simulations of the bolt - as presented in Fig. 5, all elements (bolt, washers and nut) are defined as separate parts to obtain the proper length of the shank. Tie constraints between the nut, head of the bolt and washer were used. Regarding the interaction, a normal contact between the surface of the nut and the shank is defined while the tangential behaviour is assigned as frictionless. C3D8I and C3D8R elements were used to model the bolts. However, after comparing the stresses and strains to the true stress-true strain curve defined to model plasticity, it has been concluded that element C3D8R replicates better the behaviour of the bolt failure in terms of stress and strains, while C3D8I yields slightly overestimated results. Nevertheless, the difference between the experimental and numerical simulation is negligible as Fig. 6 depicts. The reason is the axial connector, which is entirely responsible for the tensile failure.

\subsection{Mesh and finite element model}

Several solid elements were selected for a mesh sensitivity test such that the type of finite element, the size and the mesh can be assigned on the joint model. As specified in [3], the choice for the finite element largely depends on the nature of the problem. For beam-tocolumn steel joints mostly C3D8R (as adopted in [8]) or C3D8I (preferred in [9]) elements are used. In this case, local buckling of the flanges occurs, which is a problem subjected to bending, with large distortions and nonlinearities. Consequently, the following elements were considered for calibration: C3D8, C3D8R, C3D8I, C3D20R. The first element is a solid, brick element, with 8 nodes and full integration method, while the second one is 
provided with reduced integration method. The difference is mainly in the integration points -1 internal for C3D8R and 8 for C3D8. C3D8I element has incompatible modes, being an improved version of the C3D8 element. Besides the 8 integration points it has another 13 internal points which allow for an additional degree of freedom so that the parasitic shear-stress can be eliminated to model accurately bending problems. The last one - C3D20R - is a second order (quadratic) solid element while the first 3 were first order (linear) elements. The difference is the interpolation functions used. The element is provided with 20 nodes and reduced integration method ( 8 points).

Imperfections needed to be assigned on the model. It was preferred to take them into account from a buckling analysis, with different buckling modes and scale factors. The detailed procedure is presented in chapter 2.5. Consequently, buckling analyses were performed for each element type, considering from 2 up to 6 elements on the thickness of the beam flange and web. It may be concluded that the shape of the buckling modes remains the same for all models. However, the value of the Eigen mode differs, being influenced by the properties of each finite element. For C3D8, owing to the problems with shear and volumetric locking, when increasing the number of elements on thickness, the accuracy decreased. For the element with incompatible modes, it has been proven that increasing the number of elements does not increase accuracy, the difference is less than $1 \%$. However, for C3D8R, the accuracy increased very much, reaching $1 \%$ error (6 elements on thickness) with respect to the model with C3D8I, with 2 elements on thickness. In the case of the last element, C3D20R, the accuracy of the result did not increase when increasing the number of elements. The error in all cases was less than $1 \%$ with respect to the model with incompatible modes, with 2 elements on thickness.

Based on these results, the following models have been investigated further on:

- C3D8I with 2 elements on thickness

- C3D8 with 2 elements on thickness

- C3D8R with 4 elements on thickness

- C3D20R with 2 elements on thickness

In Fig. 7 is presented the influence of the finite element type upon the numerical simulation of a joint. The results are compared with the ones from the experimental test. The same element was used also for the buckling analysis with different modes - the difference is visible at the maximum force - but the scaling factors are the same.

What can be concluded from Fig. 7 and Fig. 8 is the fact that C3D8R element is the most appropriate, considering also the analysis time. The model is 10 to $20 \%$ more efficient than the one with C3D8I as Fig. 8 presents, depending on how coarse the mesh is defined in the regions considered to be elastic. As it is described in [3], C3D8I is very good in bending and large distortions, but unrealistic residual stresses can appear when unloading if the geometry has been subjected to complex deformation - the case of local buckling.

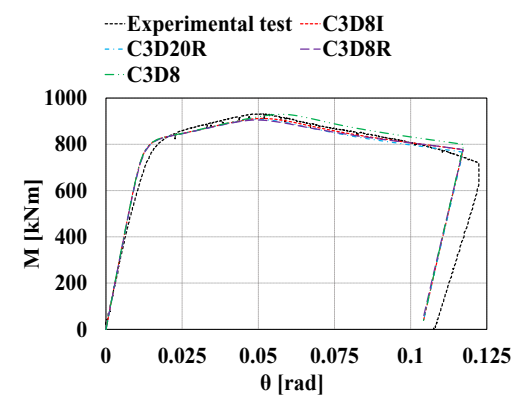

Fig. 7. Influence of finite element for the calibration of numerical model

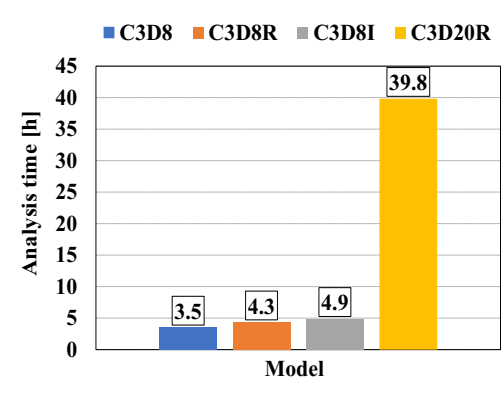

Fig. 8. Analysis time for models with different finite elements 
In Fig. 9 is presented the Equivalent Von Misses stress for the beam in the last step before unloading for C3D8I, and for C3D8R in Fig. 10. In both figures a vertical stiffener, which isolates the right part of the beam is not shown, being the reason for small stress represented with blue in the right side of the beam.

Even though a complex state of compression is present where distortion occurs, there is a big difference from 670 to $1096 \mathrm{MPa}$, adding the fact that $745 \mathrm{MPa}$ is the maximum value of the true stress defined for the beam plasticity. Moreover, after unloading, Equivalent Von Misses stress reaches almost $1600 \mathrm{MPa}$ in local regions in the model with C3D8I element for the beam.

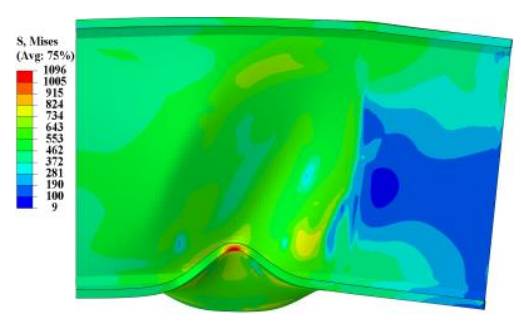

Fig. 9. Maximum Equivalent Von Misses stress for model with C3D8I

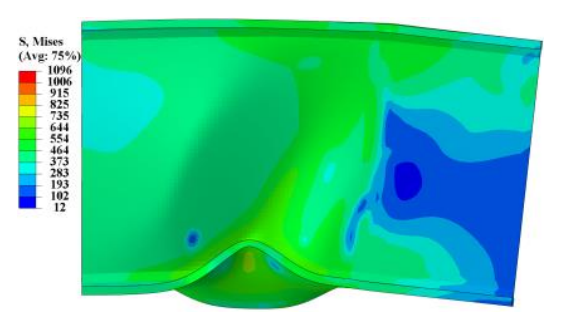

Fig. 10. Maximum Equivalent Von Misses stress for model with C3D8R

\subsection{Imperfections}

According to the design codes that are used for civil engineering, imperfections should be considered function of the type of analysis performed $[4,10]$. In detail, imperfections may be geometric or due to residual stresses. In the present study, only the geometric imperfections were investigated. A geometrically and materially nonlinear analysis with imperfections included (GMNIA) was considered. The imperfections were assigned to the beam, from a buckling analysis, using the relevant modes of vibration to obtain the shape presented in Fig. 11. Consequently, mode 1 - Fig. 12 - with negative scale factor and mode 3 - Fig. 13 - with positive scale factor were used to model imperfections such that the shape presented may be obtained. According to [11], the fabrication tolerances -accounted as imperfections, representing the sum of the relative displacements $\mathrm{k}$ and $\mathrm{k}$ ' should not exceed $0.02 \cdot \mathrm{b}$ or $6.5 \mathrm{~mm}$. In Fig. 14 and Fig. 15 is presented the influence of imperfections upon two distinct models. In may be inferred that for the first model, the best result is with $25 \%$ imperfections (expressed in function of $0.02 \cdot \mathrm{b}$ ) and for the second model, with $5 \%$ imperfections.

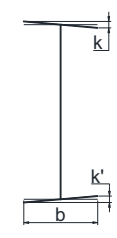

Fig. 11. Tolerances for hot rolled beams

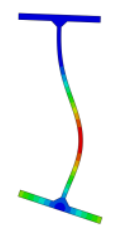

Fig. 12. Beam section Fig. 13. Beam section representing $1^{\text {st }}$ buckling mode representing $3^{\text {rd }}$ buckling mode

From the measurements of the beam, taken before testing the specimens, an average imperfection of $42 \%$ came out. However, the results are very scattered, ranging from $0 \%$ to 
more than $100 \%$. This confirms how subjective the imperfections may be and the fabrication process may influence a lot the quality of elements.

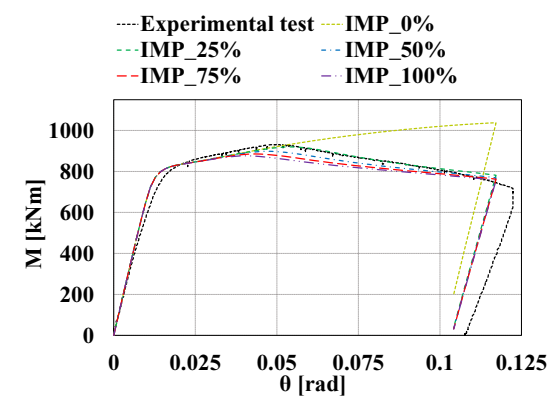

Fig. 14. Imperfection calibration for EH2_TS35 model

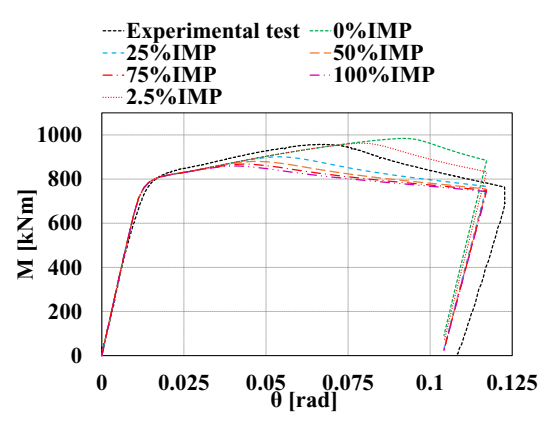

Fig. 15. Imperfection calibration for EH2_TS45 model

\section{Calibrated numerical model}

Based on the results obtained from the study presented above, a FE model has been calibrated. As finite element, C3D8R was chosen for all parts in the joint. The mesh used for the model is presented Fig. 16. It may be observed that a coarser mesh was used for the beam, outside the plastic zone in order to reduce the computational time of the analysis. An imperfection factor of $20 \%$ produced the best results and the moment-rotation curve is presented in Fig. 17.

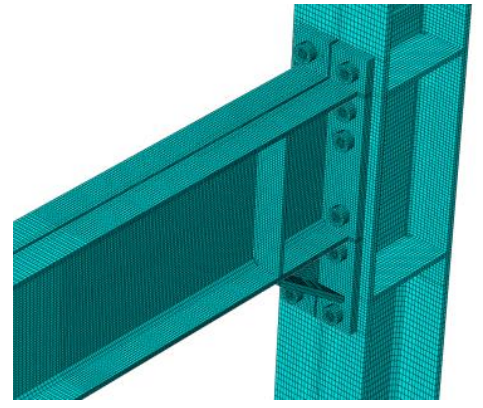

Fig. 16. Mesh of the calibrated model

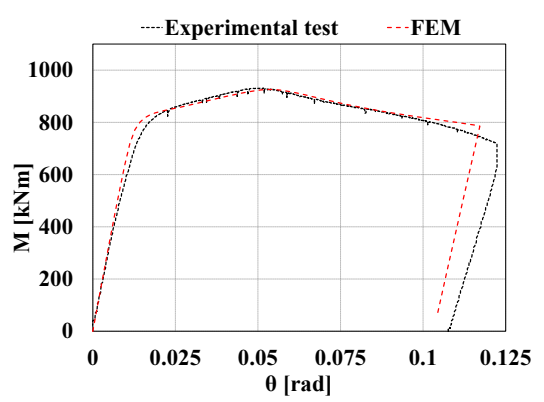

Fig. 17. M- $\theta$ curve for calibrated model

To ensure the fact that the model replicates the experimental test, the failure mode was also compared. Fig. 17 a) presents the FE model, while Fig. 17 b) the experimental test.

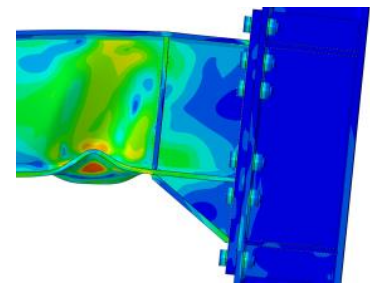

a)

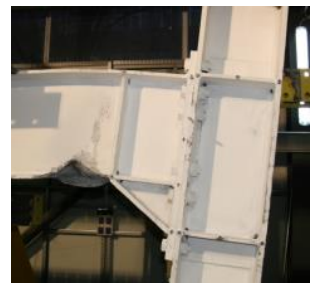

b)

Fig. 17. Failure mode in numerical model vs. experimental test 


\section{Conclusions}

The calibration of a numerical model that can replicate the behaviour of the steel joint was presented in the current paper. Specific details about the material and bolt model were outlined. In addition, the influence of the type of finite element, mesh and imperfections were presented from the results of a thorough parametric study.

It may be inferred that for the material, when there are differences between $R_{\mathrm{p} 0.2}$ and $R_{\mathrm{eH}}$, an average yield limit may be considered. For the finite element, the first order C3D8R element is appropriate in case of bending with large distortions as the case of the present application. However, a larger number of elements need to be assigned on the thickness of the element susceptible for stability loss, 4 in this case for the beam, and fewer elements in other regions where plastic failure mechanism does not occur. A factor of $20 \%$ was used for imperfection which is much smaller than fabrication tolerances.

The current model will be used in future investigation, to find specific details which can only be determined through numerical simulations. In addition, the model will be adapted such that the results with cyclic loading protocol can be trustworthy.

The research leading to these results has been funded from the European Community's Research Fund for Coal and Steel (RFCS) under grant agreement no 754048 RFCS-2016/RFCS-2016 EQUALJOINTS - PLUS. This support is gratefully acknowledged.

\section{References}

1. European pre-QUALified steel JOINTS - EQUALJOINTS, RFSR-CT-2013-00021, project website: http://dist.dip.unina.it/2013/12/09/equaljoints/

2. A. Stratan, C. Maris, D. Dubina, C. Neagu, Experimental prequalification of bolted extended end plate beam to column connections with haunches, EUROSTEEL (2017)

3. Abaqus Analysis User's Manual, Dassault Systèmes Simulia Corp., Providence, RI, USA

4. EN 1993-1-5 Eurocode 3: Design of steel structures - Part 1-5: General rules - Plated structural elements, (2005)

5. ISO 898-1: Mechanical properties of fasteners made of carbon steel and alloy steel Part 1: Bolts, screws and studs with specified property classes - coarse thread and fine pitch thread. International Standard, (2009)

6. M. Pavlović, C. Heistermann, M. Veljković, D. Pak, M. Feldmann, C. Rebelo, L. da Silva, Connections in towers for wind converters, part I: Evaluation of down-scaled experiments, J.C.S.R, 115, 445-457, (2015)

7. Y. Hu, L. Shen, S. Nie, B. Yanga, W. Sha, FE simulation and experimental tests of high-strength structural bolts under tension, J.C.S.R, 126, 174-186, (2016)

8. M. D'Aniello, D. Cassiano, R. Landolfo, Simplified criteria for finite element modelling of European preloadable bolts, S.C.S, 24, 643-658, (2017)

9. M. D'Aniello, R. Tartaglia, S. Costanzo, R. Landolfo, Seismic design of extended stiffened end-plate joints in the framework of Eurocodes, J.C.S.R, 128, 512-527, (2017)

10. EN 1993-1-1: Eurocode 3: Design of steel structures - Part 1-1: General rules, (2005)

11. EN 10034: Structural steel I and H sections, (1993) 